\title{
Clinical, imaging, and immunohistochemical characteristics of focal cortical dysplasia Type II extratemporal epilepsies in children: analyses of an institutional case series
}

\author{
Friederike Knerlich-Lukoschus, MD, PhD, ${ }^{1-3}$ Mary B. Connolly, MB, FRCP(C), ${ }^{4}$ \\ Glenda Hendson, MBBCh, FRCPC, ${ }^{5}$ Paul Steinbok, MBBS, FRCSC, ${ }^{2,3}$ and \\ Christopher Dunham, MD, FRCPC ${ }^{5}$
}

\begin{abstract}
'Department of Neurosurgery, University Hospital of Schleswig-Holstein Campus Kiel, Germany; and Divisions of ${ }^{2}$ Pediatric Neurosurgery and ${ }^{4}$ Pediatric Neurology, Department of Pediatrics, ${ }^{5}$ Division of Anatomical Pathology, Department of Pathology and Laboratory Medicine, and 'Department of Neurosurgery, British Columbia Children's Hospital, University of British Columbia, Vancouver, Canada
\end{abstract}

OBJECTIVE Focal cortical dysplasia (FCD) Type II is divided into 2 subgroups based on the absence (IIA) or presence (IIB) of balloon cells. In particular, extratemporal FCD Type IIA and IIB is not completely understood in terms of clinical, imaging, biological, and neuropathological differences. The aim of the authors was to analyze distinctions between these 2 formal entities and address clinical, MRI, and immunohistochemical features of extratemporal epilepsies in children.

METHODS Cases formerly classified as Palmini FCD Type II nontemporal epilepsies were identified through the prospectively maintained epilepsy database at the British Columbia Children's Hospital in Vancouver, Canada. Clinical data, including age of seizure onset, age at surgery, seizure type(s) and frequency, affected brain region(s), intraoperative electrocorticographic findings, and outcome defined by Engel's classification were obtained for each patient. Preoperative and postoperative MRI results were reevaluated. H \& E-stained tissue sections were reevaluated by using the 2011 International League Against Epilepsy classification system and additional immunostaining for standard cellular markers (neuronal nuclei, neurofilament, glial fibrillary acidic protein, CD68). Two additional established markers of pathology in epilepsy resection, namely, CD34 and a-B crystallin, were applied.

RESULTS Seven nontemporal FCD Type IIA and 7 Type B cases were included. Patients with FCD Type IIA presented with an earlier age of epilepsy onset and slightly better Engel outcome. Radiology distinguished FCD Types IIA and IIB, in that Type IIB presented more frequently with characteristic cortical alterations. Nonphosphorylated neurofilament protein staining confirmed dysplastic cells in dyslaminated areas. The white-gray matter junction was focally blurred in patients with FCD Type IIB. a-B crystallin highlighted glial cells in the white matter and subpial layer with either of the 2 FCD Type II subtypes and balloon cells in patients with FCD Type IIB. $a-B$ crystallin positivity proved to be a valuable tool for confirming the histological diagnosis of FCD Type IIB in specimens with rare balloon cells or difficult section orientation. Distinct nonendothelial cellular CD34 staining was found exclusively in tissue from patients with MRI-positive FCD Type IIB.

CONCLUSIONS Extratemporal FCD Types IIA and IIB in the pediatric age group exhibited imaging and immunohistochemical characteristics; cellular immunoreactivity to CD34 emerged as an especially potential surrogate marker for lesional FCD Type IIB, providing additional evidence that FCD Types IIA and IIB might differ in their etiology and biology. Although the sample number in this study was small, the results further support the theory that postoperative outcomedefined by Engel's classification-is multifactorial and determined by not only histology but also the extent of the initial lesion, its location in eloquent areas, intraoperative electrocorticographic findings, and achieved resection grade.

https://thejns.org/doi/abs/10.3171/2016.8.PEDS1686

KEY WORDS focal cortical dysplasia; intractable seizures; immunohistochemistry; a-B crystallin; CD34; surgical outcome; epilepsy

ABBREVIATIONS ABC = a-B crystallin; $\mathrm{BCCH}=$ British Columbia Children's Hospital; DRFE = early-onset drug-resistant focal epilepsy; ECoG = electrocorticography; FCD = focal cortical dysplasia; GFAP = glial fibrillary acidic protein; ILAE = International League Against Epilepsy; LFB = Luxol fast blue; NFP = nonphosphorylated neurofilament protein; NeuN = neuronal nuclei.

SUBMITTED February 29, 2016. ACCEPTED August 26, 2016.

INCLUDE WHEN CITING Published online November 25, 2016; DOI: 10.3171/2016.8.PEDS1686. 
$\mathrm{F}$ OCAL cortical dysplasia (FCD) is increasingly recognized as a major underlying neuropathological condition in children with early-onset drug-resistant focal epilepsy (DRFE). ${ }^{19,29,31}$ The term FCD subsumes a composite group of malformations of cortical development, which results from disrupted neuronal proliferation, maturation, and migration during the specific fetal developmental stages of the hexalaminar cortical structure. ${ }^{1,10}$ The causal association between FCD and epilepsy has been well established by experimental and clinical data ${ }^{2,28}$, 34,38; however, the underlying pathophysiology and molecular processes have been difficult to assess and are still under investigation. As a consequence, mechanism-driven treatment options for DRFE remain elusive to date. Drug treatment commonly proves ineffective, which leaves tailored cortical resection, with all the potential risks of brain surgical procedures, the only curative option. Fauser et al. ${ }^{15}$ recently reported favorable long-term surgical outcomes of patients with FCD; 7 years after surgery, $60 \%$ of their patients remained at Engel Class $\mathrm{I}^{13}$

Various classification schemes have been established to better characterize these highly epileptogenic lesions of disturbed cortical anatomy, and different kinds of FCD have thereby been defined based on pathological and clinical criteria. The classification system introduced by Palmini et al. ${ }^{33}$ was most widely used in the literature until its recent revision by an ad hoc task force of the International League Against Epilepsy (ILAE). ${ }^{7}$ Based on these classification systems, FCD is divided into 3 different categories that address lamination disturbances and cytoarchitectural/cellular dysplasia (FCD Types I and II) and, in the new classification system, FCD associated with principal pathologies (FCD Type III).

According to the recent clinicopathological spectrum of FCD, FCD Type II is presently considered the most severe form of FCD. ${ }^{38}$ This histopathological subgroup is defined by the presence of dysmorphic neurons (FCD Types IIA and IIB). The additional presence of balloon cells is the neuropathological hallmark of FCD Type IIB. ${ }^{6,7,33}$ Although these cytological varieties indicate biological differences between these 2 FCD Type II subtypes, no associated distinctions in clinical and imaging findings or postsurgical outcome have been identified consistent$1 y .{ }^{38}$ There are hints in the literature that temporally located FCD differs from FCD in extratemporal locations in respect to the distribution of histological subgroups, different outcomes, potentially different neuropathogeneses, and staining patterns of CD34., ${ }^{41,16,17}$ Regarding the staining patterns, only a few studies have addressed CD34 in FCD epilepsies; they provided inconsistent or even contradictory observations regarding CD34 expression in specimens from patients with FCD Type IIA or IIB (discussed in more detail in Discussion). ${ }^{11,14,30,39}$

The purpose of our investigations was to analyze our series of children with FCD Type IIA or IIB to address clinical, imaging, and histochemical aspects of the disease. By focusing on 1 type of FCD, excluding that in extratemporal locations for the reasons mentioned earlier, we intended to provide consistent information on some open questions.

Clinical and neuroimaging (MRI) features of histologi- cally confirmed nontemporal FCD Type II (reconfirmed according to the 2011 ILAE classification system) in pediatric patients who underwent tailored cortical resection for DRFE in the British Columbia Children's Hospital $(\mathrm{BCCH})$ in Vancouver, Canada, were analyzed in our studies. Additional histochemical and immunohistochemical analyses using standard cellular marker antibodies (neuronal nuclei [NeuN], neurofilament, glial fibrillary acidic protein [GFAP], CD68) were performed. Because CD34 has been suggested to be a "valuable marker for the diagnostic evaluation of neoplastic and/or malformative pathological changes in epilepsy patients," 4 we also included immunohistochemical CD34 analyses in our studies to investigate its potential diagnostic value as a distinct marker for FCD Type II subgroups. This surface glycoprotein is expressed among hematopoietic progenitor cells of myeloid precursors and vascular endothelia and during early brain development, ${ }^{8,26}$ and it therefore is also used as an oncofetal marker protein for developmental alterations and undifferentiated neoplasms. ${ }^{4}$

Last, immunohistochemical analyses of $\alpha$-B crystallin (ABC) expression, a heat shock protein that has been touted as a tissue marker of epileptic foci, ${ }^{37}$ were included in our studies to test if this marker provides additional clues for distinguishing FCD Types IIA and IIB.

\section{Methods}

Ethical review was performed by the Clinical Research Ethics Board of BCCH.

\section{Identification of Study Groups and Clinical Data Acquisition}

The prospectively maintained electronic pediatric neurosurgery and epilepsy databases of $\mathrm{BCCH}$ were queried for all patients younger than 18 years who underwent surgery for extratemporal epilepsy between 2000 and 2014. Pathology reports were searched for key words, including dyslamination, balloon cells, and homogenous eosinophilic cell bodies, and other descriptive hints for FCD. The histopathology of the identified cases was then reevaluated to confirm FCD (ambiguous cases were considered and reevaluated also). For the purpose of our study, patients were selected only if they underwent surgery for treatment-resistant epilepsy and had a histologically confirmed diagnosis of FCD Type IIA or B after repeat histopathological review.

Their charts were reviewed retrospectively to gather information on age, sex, type of seizures, seizure onset, side of resection, clinical course, and outcome, which was classified according to the Engel classification of epilepsy surgery outcome. Class I indicates that the patient is free of disabling seizures and has been completely seizure free since surgery (A), has had only nondisabling, simple, partial seizures since surgery (B), or has had some disabling seizures after surgery but has been free of disabling seizures for at least 2 years (C). Class II indicates that the patient has rare disabling seizures and was initially free of disabling seizures but now has rare seizures (A), has had rare disabling seizures since surgery (B), has had more than rare disabling seizures since surgery but rare seizures 
for the past 2 years $(\mathrm{C})$, or has had only nocturnal seizures (D). Class III indicates that the patient has had worthwhile improvement and worthwhile seizure reduction (A) or prolonged seizure-free intervals amounting to greater than half the followed-up period but not $<2$ years (B). Class IV indicates that the patient has experienced no worthwhile improvement and has had either significant seizure reduction (A) or no appreciable change (B) or the seizures have become worse (C). ${ }^{13,44}$

\section{MRI Reevaluation}

Preoperative 1.5- and 3-T MRI scans of the identified patients with FCD Type II were reevaluated blindly in digital form for FCD Type II characteristics such as cortical thickening, blurring of the gray-white matter junction, and hyperintense signal. ${ }^{29}$ High-resolution imaging protocols included 3D T1-weighted volumetric acquisition, axial and coronal 3- or 2-mm T2-weighted images, FLAIR sequences, and axial T1-weighted postcontrast imaging. In addition, postoperative scans were reevaluated to determine the correlation of resected cortical areas with the preoperatively identified lesions. Results were reconfirmed with a pediatric neuroradiologist.

\section{Histochemistry and Immunohistochemistry}

The resected tissues were processed in a routine manner for light microscopy. Four-micrometer-thick sections were cut from the formalin-fixed and paraffin-embedded blocks. All microscopic slides were prepared using standard automated techniques. All of the original stains from our FCD Type II cases were reviewed and supplemented if not used at the time of original reporting; these stains included H \& E, H \& E/Luxol fast blue (LFB), and Cresyl Violet/LFB. Cases were reevaluated using the 2011 ILAE classification system, and only instances of extratemporal FCD Type IIA or B were included in the study.

Newly reevaluated FCD Type IIA and IIB cases were then assessed further by immunohistochemical staining with established antibodies, including NeuN (MAB377, Chemicon; 1:400 dilution), nonphosphorylated neurofilament protein (NFP) (SMI-311R, Cedarlane-Covance; 1:500 dilution), GFAP (astroglial marker; 760-4345, Ventana; prediluted), CD68 (macrophage/activated microglia; 790-2931, Ventana; prediluted), CD34 (endothelial marker and abnormal highly ramifying cell highlighter in some epilepsy specimens; 790-2927, Ventana; prediluted), and ABC (marks among other balloon cells of FCD Type IIB; NCL-ABC-512, Leica; 1:100 dilution). For immunohistochemical slide preparation, we used the Ventana BenchMark XT autostainer (Ventana Medical Systems) and the iVIEW universal DAB detection kit.

\section{Results}

We identified 14 patients with extratemporal FCD Type II who underwent surgery at BCCH between 2000 and 2014. Applying the 2011 ILAE classification system on $\mathrm{H}$ \& E-stained sections obtained during tailored cortical resection, 7 patients met the criteria for FCD IIA, and 7 met the criteria for FCD IIB (Table 1).

\section{Clinical Characteristics}

Clinical characteristics of patients included in this study are summarized in Table 2 . The female/male ratios were 5:2 in patients with FCD IIA and 4:3 in those with FCD Type IIB. The mean ages of patients at epilepsy onset were 42 months (range 3 months to 7 years) in the FCD Type IIA group and 66 months (range 2 years to 11 years 2 months) in the FCD Type IIB group. There were no gross differences in seizure type or frequency between these 2 groups.

The mean ages of patients at surgery (first resection) were 8.7 years (range $2-15$ years) in the FCD Type IIA group and 10.7 years (range 4.2-16 years) in the FCD Type IIB group.

In patients with FCD IIA, resected lesions were located in the frontal $(n=3)$ and occipital $(n=4)$ brain regions. In patients with FCD Type IIB, lesions were found in the frontal $(n=4)$, occipital $(n=1)$, and frontal/parietal lobes $(\mathrm{n}=2)$. Three of 7 patients with FCD Type IIA underwent 2 resections, whereas 4 of 7 with FCD Type IIB underwent more than 1 resection (1 patient underwent 3 resections, and 3 patients underwent 2 resections).

Surgical outcomes in patients after cortical resection for FCD Type IIA were Engel Classes IA $(n=5)$, IIIA ( $n=$ $1)$, and IID $(\mathrm{n}=1)$ and for those with FCD Type IIB were Engel Classes IA $(n=4)$, IIIA $(n=1)$, and IVA $(n=2)$.

The mean follow-up durations were 4.3 years (range $2-7$ years) for the FCD Type IIA group and 4.7 years (range 14 months to 9.5 years) for the FCD Type IIB group.

\section{Intraoperative Electrocorticography}

In every case, tailored cortical resection was performed with intraoperative electrocorticography (ECoG) and after extracranial monitoring with subdural grids (Cases 3 , $6,8,9,10$, and 14). In Cases 4, 7, 10, and 14, complete excision of the epileptic focus was not achievable. Although postresection ECoG in Case 7 revealed significant reduced activity in the resected frontal area (main epileptic focus), there were still residual spikes in the adjacent motor strip that was left intact. In Case 10, mesial postresection ECoG was technically difficult because of anatomical conditions, and complete resection of the areas affected by FCD was probably not achieved. In Cases 4 and 14, only partial resection was feasible because of widespread cortical and subcortical lesions (detailed in the following section), and residual epileptiform activity was seen with postresection ECoG.

\section{MRI Findings}

FCD Type IIA

In 3 of the FCD IIA cases, similar distinct cortical lesions were seen on preoperative MRI (Table 1 and Fig. 1). In Case 1, there was an area of gray-white matter interface blurring at the posterior aspect of the right calcarine sulcus (seen only on 3-T MRI). This blurring was best identified on the 3D T1-weighted images (Fig. 1A). On coronal T2-weighted images, there was a corresponding fine band of hyperintensity at the interface between the gray and white matter. Appearances were in keeping with an area of FCD. Case 5 had an area of thickened cortex with slight 
TABLE 1. FCD Type IIA and IIB cases*

\begin{tabular}{|c|c|c|c|c|c|}
\hline $\begin{array}{l}\text { Case } \\
\text { No. }\end{array}$ & Sex & $\begin{array}{l}\text { FCD } \\
\text { Type }\end{array}$ & Epileptic Focus $†$ & $\begin{array}{c}\text { MRI } \\
\text { Resultf }\end{array}$ & MRI Details \\
\hline 1 & $\mathrm{~F}$ & $\| A$ & Occipital rt & Lesion & $\begin{array}{l}\text { Blurring of } g / w \text { best identified on 3D T1-weighted images; fine band of hyperintensity at } \\
\text { g/w in rt calcarine sulcus on T2-weighted images }\end{array}$ \\
\hline 3 & $\mathrm{~F}$ & $\| A$ & Frontal It & Negative & \\
\hline 5 & M & IIA & Frontal It & Lesion & Area of thickened cortex w/ burring of $\mathrm{g} / \mathrm{w}$ in It frontal lobe \\
\hline 6 & $\mathrm{~F}$ & $\| \mathrm{A}$ & Occipital It & Negative & \\
\hline 8 & $\mathrm{~F}$ & IIA & Occipital It & Lesion & Abnormal loss of definition of $\mathrm{g} / \mathrm{w}$ of the It parietooccipital sulcus \\
\hline 9 & M & $\| A$ & Occipital rt & Negative & \\
\hline 2 & $M$ & IIB & Frontal rt & Lesion & $\begin{array}{l}\text { T2-weighted hyperintensity w/ cortical blurring \& transmantle sign in rt frontal lobe in the- } \\
\text { anterior superior frontal gyrus }\end{array}$ \\
\hline 4 & $F$ & IIB & Frontal/parietal It & Lesion & $\begin{array}{l}\text { Multilobar hyperintense T2-weighted \& FLAIR signals; indistinct g/w; additional hyperin- } \\
\text { tense T2-weighted and FLAIR signal in the anterior limb \& genu of the It internal capsule }\end{array}$ \\
\hline 13 & $\mathrm{~F}$ & IIB & Frontal rt & Lesion & $\begin{array}{l}\text { Focal area of abnormal g/w blurring in the anteromedial rt frontal cortex (on 3D T1- \& T2- } \\
\text { weighted \& FLAIR images) w/ transmantle sign directed toward the frontal horn of the rt } \\
\text { lateral ventricle; overall size of the affected location was } \sim 1 \mathrm{~cm} \text { in diameter, at the most }\end{array}$ \\
\hline 14 & $\mathrm{~F}$ & IIB & Occipital rt & Lesion & $\begin{array}{l}\text { Subtle cortical hyperintensity in the rt occipital lobe below the calcarine fissure on T2- } \\
\text { weighted images; blurring of the g/w relatively widespread w/in the inferior rt occipital } \\
\text { lobe, w/ a diameter of } \sim \geq 2.5 \mathrm{~cm}\end{array}$ \\
\hline 10 & M & $\| \mathrm{B}$ & $\begin{array}{l}\text { Rt precentral \& post- } \\
\text { central gyrus }\end{array}$ & Negative & $\begin{array}{l}\text { No cortical abnormality/no cortical thickening; only hyperintense spots in frontal subcorti- } \\
\text { cal WM }\end{array}$ \\
\hline
\end{tabular}

$\mathrm{g} / \mathrm{w}=$ gray-white matter interface; $\mathrm{WM}=$ white matter.

* FCD Type IIA (Cases 1, 3, and 5-9): $n=7$ (5 female and 2 male; epileptic focus, 3 frontal and 4 occipital; 3 with lesion evident on MR images [cortical dysplasia] and 4 with no lesions seen on MR images [negative]); FCD Type IIB (Cases 2, 4, and 10-14): $n=7$ (4 female and 3 male; epileptic focus, 4 frontal, 1 occipital, and 2 frontal/ parietal; 6 with cortical lesion seen on MR images and 1 with no lesions seen on MR images).

$\dagger$ According to electroencephalography/ECoG results.

$\ddagger \mathrm{MRI}$ findings in respect to lesions typical for cortical dysplasia.

blurring on T2-weighted images between the cortex and white matter in the left frontal cortical area, anterior to the motor strip (Fig. 1B). Similar changes were seen in Case 8 , in which abnormal loss of the defined gray-white matter interface in the left parieto-occipital sulcus was seen with 3-T imaging (Fig. 1C).

The other 4 patients with FCD Type IIA remained lesion free on evaluation of repeated consecutive 1.5- and 3-T MRI.

\section{FCD Type IIB}

Focal cortical and associated white matter changes were seen in 6 of 7 patients with FCD Type IIB. In Case 2, an area of mild focal hyperintensity with cortical blurring and a small white matter tail in the right frontal lobe in the midanterior superior frontal gyrus tapering toward the ventricle ("transmantle sign") was found on T2-weighted and FLAIR images (Fig. 1D). In Case 4, a hyperintense signal and blurring of the cortical-white matter junction in the left frontal cortex, involving parts of the insular cortex and parietal cortex, extending into the white matter were seen on T2-weighted/FLAIR images (Fig. 1E and F). Furthermore, a focal region of hyperintensity in the anterior limb and genu of the left internal capsule and tha- lamic involvement were found on a T2-weighted/FLAIR image (Fig. 1F).

In Case 11, we found a small focus in the medial aspect of the right frontal lobe anterior to the central gyrus with cortical blurring on T2-weighted images and slight increased signal intensity on FLAIR images (Fig. 1G). In Case 13, a focal area of gray matter blurring in the anteromedial right frontal cortex, best seen on 3D T1-weighted and thin-section T2-weighted and FLAIR images, was seen along with a tail of ill-defined hyperintensity directed toward the superolateral margin of the frontal horn of the right ventricle (Fig. 1H and I). This area of abnormality was located just anterolateral to the superior frontal sulcus. The overall size of the affected location was approximately $1 \mathrm{~cm}$ in diameter. In addition, ictal SPECT confirmed increased perfusion in this area. Hyperintensity in a small area of the right frontal cortex immediately above the insula, just anterior to the central sulcus, with minimal cortical blurring was seen on T2-weighted/ FLAIR sequences in Case 12 (Fig. 1J).

In Case 14, there was a relatively widespread lesion within the inferior right occipital lobe with a diameter of approximately $2.5 \mathrm{~cm}$ or greater. Subtle cortical hyperintensity with loss of subcortical hypointensity of myelina- 
TABLE 2. Clinical characteristics of FCD Type IIA and IIB cases under investigation*

\begin{tabular}{|c|c|c|c|c|c|c|c|c|}
\hline $\begin{array}{l}\text { Case } \\
\text { No. }\end{array}$ & Sex & Onset Age & Seizure Type & Seizure Frequency & $\begin{array}{l}\text { Age at } \\
\text { Surgery† }\end{array}$ & Surgery Site & $\begin{array}{l}\text { Outcome } \\
\text { (Engel } \\
\text { Class) }\end{array}$ & Follow-Up \\
\hline 1 & $\mathrm{~F}$ & 7 yrs & $\begin{array}{l}\text { Focal dyscognitive; focal } \\
\text { evolving to generalized } \\
\text { seizures }\end{array}$ & $\begin{array}{l}\text { Monthly clinical sei- } \\
\text { zures; daily electro- } \\
\text { graphic seizures }\end{array}$ & $10 \mathrm{yrs}$ & $\begin{array}{l}\text { Occipital rt calcarine } \\
\text { sulcus }\end{array}$ & $\mathrm{IA}$ & 25 mos \\
\hline 3 & $\mathrm{~F}$ & $7 \mathrm{yrs}$ & Focal gelastic & 20/day & $\begin{array}{l}13 \text { yrs } 2 \text { mos, } \\
16 \text { yrs }\end{array}$ & $\begin{array}{l}\text { Frontal It; subdural grid for } \\
3 \text { days }\end{array}$ & $\mathrm{IA}$ & $38 \mathrm{mos}$ \\
\hline 5 & M & $17 \mathrm{mos}$ & Focal motor & $\begin{array}{l}\text { Daily (multiple per } \\
\text { hour) }\end{array}$ & 5 yrs & Frontal lobe & $\begin{array}{l}\text { 2D/sub- } \\
\text { clinical } \\
\text { seizure } \\
\text { only }\end{array}$ & $5 \mathrm{yrs}$ \\
\hline 6 & $\mathrm{~F}$ & $3 \mathrm{mos}$ & $\begin{array}{l}\text { Epileptic spasms; focal } \\
\text { dyscognitive }\end{array}$ & Multiple daily & $13 \mathrm{yrs}, 14 \mathrm{yrs}$ & Lt occipital & $\mathrm{IA}$ & 7 yrs \\
\hline 7 & $\mathrm{~F}$ & $1 \mathrm{mo}$ & $\begin{array}{l}\text { Focal dyscognitive, evolv- } \\
\text { ing to generalized; recur- } \\
\text { rent status epilepticus }\end{array}$ & $\begin{array}{l}\text { 2/day; status epi- } \\
\text { lepticus for } 3 \text { wks } \\
\text { before surgery }\end{array}$ & 3 yrs & Frontal rt & IIIA & 5 yrs 5 mos \\
\hline 8 & $\mathrm{~F}$ & $7 \mathrm{yrs}$ & Focal dyscognitive & Multiple daily & 15 yrs, 16 yrs & Occipital It & $\mathrm{IA}$ & 6 yrs \\
\hline 9 & M & $1 \mathrm{mo}$ & $\begin{array}{l}\text { Infantile spasms; focal } \\
\text { dyscognitive }\end{array}$ & Multiple daily & $2 \mathrm{yrs}$ & Rt occipital; subdural grids & $\mathrm{IA}$ & 3 yrs 4 mos \\
\hline 2 & M & $\begin{array}{l}11 \mathrm{yrs} \pm 2 \\
\mathrm{mos}\end{array}$ & Gelastic & 2-3/day & $11 \mathrm{yrs}$ & Frontal rt & $\mathrm{IA}$ & $38 \mathrm{mos}$ \\
\hline 4 & $\mathrm{~F}$ & $3 y$ & Focal dyscognitive & 40/day & $\begin{array}{r}3 \text { yrs } 3 \text { mos, } 3 \\
\text { yrs } 10 \text { mos, } \\
4 \text { yrs } 2 \text { mos }\end{array}$ & $\begin{array}{l}\text { Frontal/parietal It; basal } \\
\text { ganglia It }\end{array}$ & IVA & 4 yrs 5 mos \\
\hline 10 & M & 11 & $\begin{array}{l}\text { Focal; focal evolving to } \\
\text { generalized convulsive }\end{array}$ & Multiple daily & $\begin{array}{l}15 \text { yrs } 6 \text { mos, } \\
15 \text { yrs } 8 \text { mos }\end{array}$ & Frontal lobe; subdural grids & IVA & 4 yrs \\
\hline 11 & M & $2-3$ yrs & Focal & 50-60/day & 13 yrs & Rt mesial/frontal area & IA & 9 yrs 5 mos \\
\hline 12 & $\mathrm{~F}$ & 6 yrs 6 mos & Focal motor & 2-3/day & 16 yrs, 17 yrs & Rt frontal lobe & $\mathrm{IA}$ & 7 yrs 9 mos \\
\hline 13 & $\mathrm{~F}$ & $2 \mathrm{yrs}$ & Focal gelastic & $3-4 / \mathrm{mo}$ & 10 yrs 6 mos & $\begin{array}{l}\text { Rt frontal lobe (intraop } \\
\text { ECoG, depth electrodes) }\end{array}$ & $\mathrm{IA}$ & 9 yrs 3 mos \\
\hline 14 & $\mathrm{~F}$ & $2 \mathrm{yrs}$ & $\begin{array}{l}\text { Focal dyscognitive; focal } \\
\text { evolving to generalized } \\
\text { convulsive }\end{array}$ & 5-10/day & $\begin{array}{l}6 \text { yrs, } 6 \text { yrs } 10 \\
\text { mos }\end{array}$ & $\begin{array}{l}\text { Rt occipital lobe; subdural } \\
\text { grids }\end{array}$ & IIIA & $14 \mathrm{mos}$ \\
\hline
\end{tabular}

* All patients underwent ECoG-tailored surgery.

† Some patients underwent multiple surgeries.

tion in the right occipital lobe below the calcarine fissure was apparent on T2-weighted/FLAIR images (Fig. 1K).

Case 10 involved the only patient with FCD Type IIB in whom there were no cortical lesions detectable on 3-T MRI. The only findings in this case were 3 small foci of high signal intensity within the left frontal white matter ( 2 in the subcortical white matter and 1 in the deep white matter), which were seen consistently on FLAIR images (Fig. 1L shows 1 of these white matter spots). These abnormalities were considered nonspecific and not associated with epileptic foci.

\section{Postoperative MRI}

In respect to lesional MRI, resection defects in all patients were localized in the expected area of cortical dysplasia. MRI in Cases 2, 8, 11, 12, 13, and 14 revealed complete resection of the area of previously identified abnormal cortex. In Case 5, there was subtle new white matter hyperintensity near the margins of the lateral ven- tricles on T2-weighted images, but otherwise, complete resection of the area previously identified as abnormal cortex was achieved. In respect to the patient in Case 4, who presented initially with extended presurgical lesions, the resections involved frontal lobectomy, and most of the left caudate nucleus, lentiform nucleus, anterior limb, and anterior body of the corpus callosum were removed. After the final resection, no significant residual cortical signal abnormality was seen on MR images. The patient in Case 11 received only a postsurgical cranial CT in our institution to document the resection defect in the expected location of cortical dysplasia. MRI of the patient in Case 14 did not reveal any obvious hints for residual cortical dysplasia; however, there was new hyperintensity in the right optic radiation on T2-weighted images. There were no postresection MR images available in Case 1.

In respect to cases in which no lesions were found with MRI and cortical resection was tailored by intraoperative ECoG or subdural grids, resected areas involved cortical 

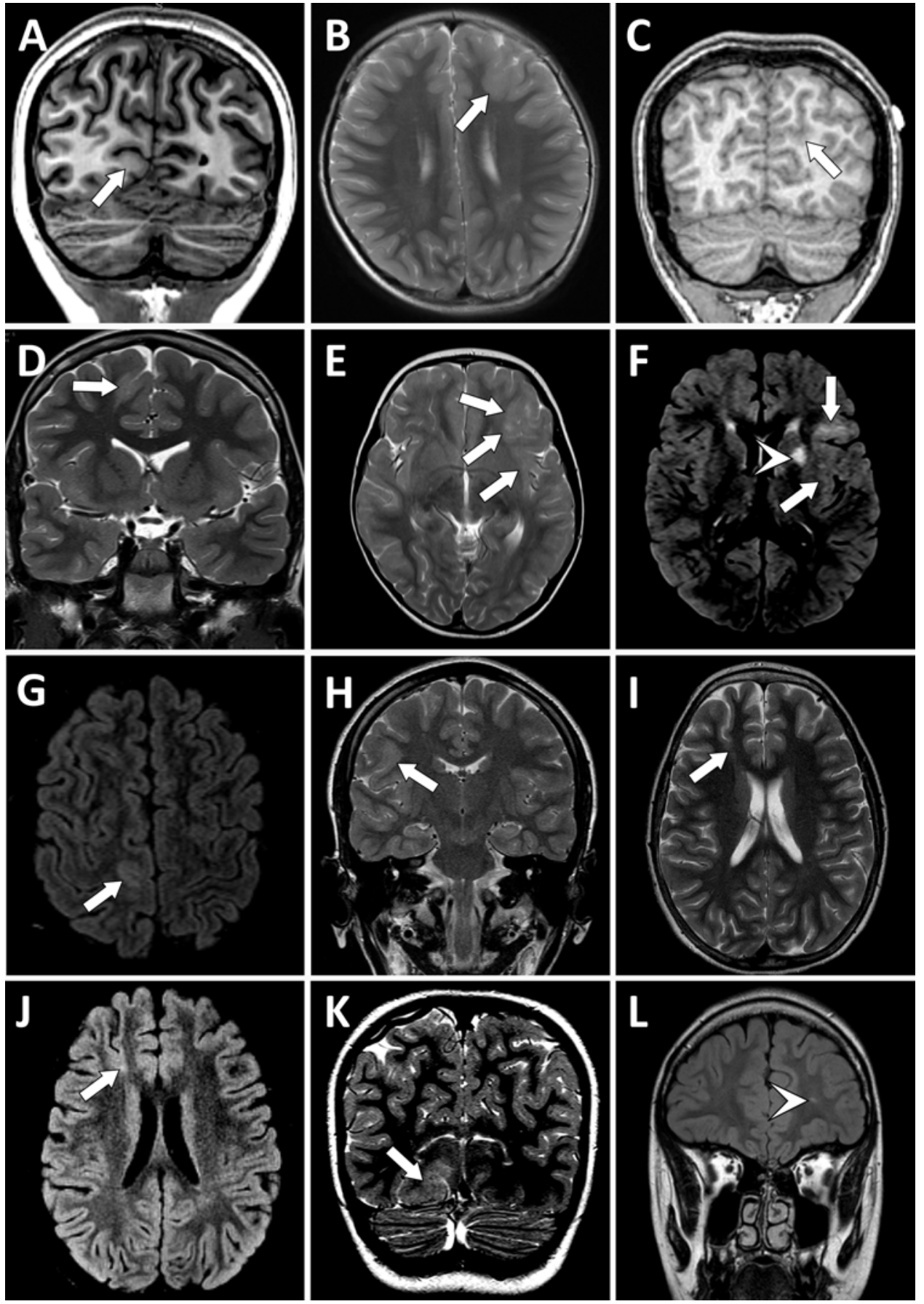

FIG. 1. Imaging features of MRI-positive FCD Type IIA (A and B) and IIB (D-L) cases. A: Case 1. Gray-white matter interface blurring (arrow) at the posterior aspect of the right calcarine sulcus on 3D T1-weighted imaging. B: Case 5. Area of thickened cortex with slight burring (arrow) in the left frontal cortical area (thin-cut T2-weighted imaging). C: Case 8. Abnormal loss of the gray-white matter interface in defined cortical area in the left parietooccipital sulcus (arrow) (3D T1-weighted imaging). D: Case 2. Focal hyperintensity (arrow) with transmantle sign in left frontal lobe on T2-weighted imaging. $\mathbf{E}$ and F: Case 4. Hyperintense signal on T2-weighted (E, arrows) and FLAIR ( $F$, arrows) images in the left frontal cortex involving parts of the insular cortex and parietal cortex and extending into the white matter plus focal FLAIR hyperintensity in the anterior limb and genu of the left internal capsule and thalamic involvement (F, arrowhead). G: Case 11. Small focus in the right frontal lobe anterior to the central gyrus with cortical blurring and slight increased signal intensity (arrow) on FLAIR image. H: Case 12. hyperintensity on T2-weighted image confined to a small area of the right frontal cortex (arrow). I and J: Case 13. Focal area of gray matter blurring in the anteromedial right frontal cortex with a tail of ill-defined hyperintensity directed toward the superolateral margin of the frontal horn of the right ventricle on T2-weighted (I, arrow) and FLAIR (J, arrow) images. K: Case 14. Hyperintense lesion within the inferior right occipital lobe (arrow) on FLAIR-weighted image. L: Case 10. Hyperintense spot in the left frontal deep white matter (arrowhead) on FLAIR image. 
TABLE 3. Histopathological findings in cases of FCD Type IIA

\begin{tabular}{|c|c|c|c|c|c|c|c|}
\hline \multirow[b]{2}{*}{$\begin{array}{l}\text { Case } \\
\text { No. }\end{array}$} & \multicolumn{7}{|c|}{ Results of Staining } \\
\hline & $H \& E$ & NeuN/Neurofilament & LFB/H \& E & $\begin{array}{l}\text { GFAP, } \\
\text { A/CG }\end{array}$ & CD68 & $\begin{array}{l}\mathrm{ABC}, \mathrm{CO} / \\
\mathrm{WM} / \mathrm{SP}\end{array}$ & $\begin{array}{l}\text { CD34, } \\
\text { CE }\end{array}$ \\
\hline 1 & $\begin{array}{l}\text { Focal dyslamination, Layers } \\
\text { III-V w/ focal increased } \\
\text { cortical thickness }\end{array}$ & $\begin{array}{l}\text { Dysmorphic, maloriented cells w/ max } \\
\text { diameter of } 59 \mu \mathrm{m}) ; \text { condensed Nissl } \\
\text { substance; no neurons in } \mathrm{g} / \mathrm{w}\end{array}$ & $\begin{array}{l}\text { No demyelination; focal poor } \\
\text { demarcation of } \mathrm{g} / \mathrm{w}\end{array}$ & $++/++$ & ++ & $+/-1-$ & - \\
\hline 3 & $\begin{array}{l}\text { Focal dyslamination, Layers } \\
\text { II-IV }\end{array}$ & $\begin{array}{l}\text { One small focus w/ dysmorphic malori- } \\
\text { ented neurons; max diameter of } 70 \\
\mu \mathrm{m} ; \text { no neurons in WM border }\end{array}$ & $\begin{array}{l}\text { No demyelination; g/w not } \\
\text { blurred }\end{array}$ & $+/++$ & + & $-1-1-$ & - \\
\hline 5 & $\begin{array}{l}\text { Focal dyslamination, all } 6 \\
\text { layers w/ increased corti- } \\
\text { cal thickness }\end{array}$ & $\begin{array}{l}\text { Dysmorphic maloriented neurons; some } \\
\text { cells up to } 100 \mu \text { m in diameter; also } \\
\text { seen focally in adjacent WM }\end{array}$ & $\begin{array}{l}\text { No demyelination; g/w fairly } \\
\text { distinct }\end{array}$ & $++/++$ & + & $+/++/+$ & - \\
\hline 6 & $\begin{array}{l}\text { Focal dyslamination; Layers } \\
\text { III and IV }\end{array}$ & $\begin{array}{l}\text { Small area of dysmorphic cells in Layer } \\
\text { III/IV; max cell diameter of } 20 \mu \mathrm{m}\end{array}$ & $\begin{array}{l}\text { No demyelination; g/w not } \\
\text { blurred }\end{array}$ & $++/++$ & ++ & $+/++/+$ & - \\
\hline 7 & $\begin{array}{l}\text { Focal area w/ dyslamination } \\
\text { throughout all layers }\end{array}$ & $\begin{array}{l}\text { Focal area w/ maloriented dysmorphic } \\
\text { cells; cell size up to } 60 \mu \mathrm{m}\end{array}$ & $\begin{array}{l}\text { No demyelination; g/w not } \\
\text { blurred }\end{array}$ & $++/++$ & + & $-/+++/+$ & - \\
\hline 8 & $\begin{array}{l}\text { Dyslamination Layers I-VI, } \\
\text { increased cortical thick- } \\
\text { ness }\end{array}$ & $\begin{array}{l}\text { Dysmorphic maloriented neurons; max } \\
\text { cell diameter of } \sim 50 \mu \mathrm{m}\end{array}$ & $\begin{array}{l}\text { No demyelination; focal blurring } \\
\text { of g/w caused by dysplastic } \\
\text { maloriented neurons }\end{array}$ & $++/++$ & ++ & $+/+++/++$ & - \\
\hline 9 & $\begin{array}{l}\text { Focal areas of dyslamina- } \\
\text { tion of all layers }\end{array}$ & $\begin{array}{l}\text { Dysmorphic neurons, scattered through } \\
\text { all cortical layers, mainly midlayers; } \\
\text { max cellular diameter of } 47 \mu \mathrm{m}\end{array}$ & $\begin{array}{l}\text { No demyelination; no g/w } \\
\text { blurring }\end{array}$ & $++/+$ & ++ & $++/+++/++$ & - \\
\hline
\end{tabular}

$\mathrm{A}=$ astrocytes; $\mathrm{BL}=$ balloon cells; $\mathrm{CE}=$ nonendothelial cellular staining; $\mathrm{CG}=$ Chaslin's gliosis; $\mathrm{CO}=$ cortex; $\mathrm{SP}=$ pial/subpial; $+=$ occasional; $++=$ frequent; $+++=$ abundant; - = no staining.

regions of identified epileptogenic zones. Postoperative MRI in Cases 3, 6, and 9 revealed resection areas anatomically located in the epileptic focus and no evidence of new pathology. The patient in Case 7 presented with a frontal resection cavity excluding the region of deep central sulcus, which had an unusual morphology, but otherwise the cortex had a normal appearance in presurgical MR images (Table 1). The patient in Case 10 presented with a minimal postoperative hyperintensity behind the resection cavity (after left frontal resection) seen on T2weighted images.

\section{Pathological Findings FCD Type IIA}

Histopathological findings are summarized in Tables 3 and 4. Figure 2 shows histochemical and immunohistochemical staining. Patients with FCD Type IIA exhibited focal dyslamination with numerous dysmorphic neurons scattered throughout all neocortical layers but more commonly in the mid- to deeper cortical layers. Neuronal staining was found helpful for confirming dyslaminated areas, which were identified on $\mathrm{H} \& \mathrm{E}$-stained sections (Fig. 2A and B, NeuN staining). These neurons exhibited an abnormal orientation (Fig. 2B and C, NeuN and neurofilament staining, respectively) and abnormally large cell bodies (approximate median diameter $58 \pm 22 \mu \mathrm{m}$ ) (Fig. 2D, Bielschowsky staining).

The gray-white matter junction in FCD Type IIA cases appeared distinct in all cases, and there were no hints of demyelination according to $\mathrm{H} \& \mathrm{E} / \mathrm{LFB}$ staining (Fig. 2E).

Glial fibrillary acidic protein staining revealed dense astrogliosis in all the patients with subpial Chaslin's gliosis (Fig. 2F, thick arrows) and starlike cells with multibranched cellular processes (Fig. 2F, which shows more superficial cortical areas, and Fig. 2G, which shows GFAP staining in deeper cortical layers).

CD68-labeled microglial cells were scattered throughout cortical (Fig. 2H) and subcortical areas of the specimens under investigation.

In all cases of FCD Type IIA, CD34 immunolabeling was confined to endothelial/vascular staining patterns (Fig. 2I); no nonendothelial cellular staining was detected in the examined specimens from patients with FCD Type IIA. This constitutive endothelial immunoreactivity to CD34 was detected in all specimens from patients with FCD, regardless of subtype, and thus provided an intrinsic positive control for the findings on nonendothelial cellular staining in specimens from patients with FCD Type IIB (described in the next section).

$\alpha-B$ crystallin immunohistochemistry revealed different staining patterns. There was dense bandlike staining in the subpial molecular layer comparable to that in GFAPmarked Chaslin's gliosis (Fig. 2J, thick arrows). Directly subjacent to this staining pattern, cortical $\mathrm{ABC}$ staining was less dense and "netlike" (Fig. 2H, arrows). There were small, round immunopositive cells scattered throughout the dyslaminated cortical areas resembling satellite glial cells (Fig. 2K, ABC-immunoreactive cells [black arrows] closed to immunonegative neuronal cells [white arrow]). Abundant $\mathrm{ABC}$ staining was found in small round cells in the subcortical white matter, which fulfills the morphological criteria for glial cells (Fig. 2L). 
TABLE 4. Histopathological findings in cases of FCD Type IIB

\begin{tabular}{|c|c|c|c|c|c|c|c|}
\hline \multirow[b]{2}{*}{$\begin{array}{l}\text { Case } \\
\text { No. }\end{array}$} & \multicolumn{7}{|c|}{ Results of Staining } \\
\hline & $H \& E$ & NeuN/Neurofilament & LFB/H \& E & $\begin{array}{l}\text { GFAP, A/ } \\
\text { CG }\end{array}$ & CD68 & $\begin{array}{c}\mathrm{ABC} \\
\mathrm{CO} / \mathrm{WM} / \mathrm{SP}\end{array}$ & $\begin{array}{l}\text { CD34, } \\
\text { CE }\end{array}$ \\
\hline 4 & $\begin{array}{l}\text { Dyslamination all layers, } \\
\text { most prominent III to VI } \\
\text { w/ cortical thickening; BL }\end{array}$ & $\begin{array}{l}\text { Dysmorphic maloriented cells; sizes } \\
\text { up to } 75 \mu \mathrm{m} \text {; mainly in deeper } \\
\text { layers \& } \mathrm{g} / \mathrm{w}\end{array}$ & $\begin{array}{l}\text { Blurring of } \mathrm{g} / \mathrm{w} \text { caused } \\
\text { by } \mathrm{BL} \& \text { dysmor- } \\
\text { phic cells }\end{array}$ & $+++/++B L$ & +++ & $++/++++/++B L$ & + \\
\hline 10 & $\begin{array}{l}\text { Dyslamination Layers II, IV, } \\
\text { \& V; BL }\end{array}$ & $\begin{array}{l}\text { Dysplastic maloriented neurons; few } \\
\text { reach sizes of } \sim 40 \mu \mathrm{m} \text { in diameter }\end{array}$ & $\begin{array}{l}\text { Grey/WM layer is } \\
\text { intact; BL are scat- } \\
\text { tered through WM }\end{array}$ & $++/+B L$ & +++ & $++/+++/+B L$ & - \\
\hline 11 & Dyslamination all layers; BL & $\begin{array}{l}\text { Some dysmorphic maloriented neu- } \\
\text { rons do not respect g/w; cell size } \\
\text { up to } 80-110 \mu \mathrm{m} \text { (diameter) }\end{array}$ & $\begin{array}{l}\text { Blurring of } \mathrm{g} / \mathrm{w} \text { caused } \\
\text { by } \mathrm{BL} \& \text { neuronal } \\
\text { cells }\end{array}$ & $++B L$ & $++/-$ & $+/+++/-B L$ & + \\
\hline 13 & $\begin{array}{l}\text { Dyslamination throughout all } \\
\text { layers; BL }\end{array}$ & $\begin{array}{l}\text { Large dysplastic neurons; max diam- } \\
\quad \text { eter } 115 \mu \mathrm{m}\end{array}$ & $\begin{array}{l}\text { Blurring of } g / w \text { caused } \\
\text { by } B L\end{array}$ & $++/++B L$ & + & $++/+++/-B L$ & + \\
\hline 14 & $\begin{array}{l}\text { Dyslamination throughout } \\
\text { all layers; especially in } \\
\text { deeper layers; BL }\end{array}$ & $\begin{array}{l}\text { Dysplastic maloriented neurons } \\
\text { throughout all layers; up to } 70 \mu \mathrm{m} ; \\
\text { also single dysplastic neuronal } \\
\text { cells scattered in the adjacent } \mathrm{g} / \mathrm{w}\end{array}$ & $\begin{array}{l}\text { Blurring of } \mathrm{g} / \mathrm{w} \text { caused } \\
\text { by } \mathrm{BL}\end{array}$ & $+++/++B L$ & +++ & $++/++++/++B L$ & + \\
\hline
\end{tabular}

\section{FCD Type IIB}

Based on H \& E histology, 7 cases of FCD Type IIB were identified with focal cortical dyslamination (Fig. 3A), dysplastic neurons scattered throughout all cortical layers (Fig. 3A-C) and partially crossing the white matter-cortical border (Fig. 3C, arrows), and balloon cells (Fig. 3D, white arrow; located close to dysplastic neurons [black arrow]).

Staining with $\mathrm{H} \& \mathrm{E} / \mathrm{LFB}$ revealed that the gray-white matter border was disturbed by intermingled balloon cells (Fig. 3E and F). Balloon cells were found in abundance, especially in the deeper cortical layers and adjacent graywhite matter border; they exhibited typical roundish morphology lacking cellular processes with abundant cytoplasm and displaced nuclei (Fig. 3D and $\mathrm{F}$ inset).

As with cases of FCD Type IIA, there was strong subpial (Fig. 3G, arrows), cortical (Fig. 3G and H), and gray-white matter junction (Fig. $3 \mathrm{H}$ ) astrogliosis seen with GFAP staining. Furthermore, balloon cells exhibited strong GFAP staining (Fig. 3I).

Staining with CD68 revealed microglial activation (Fig. $3 \mathrm{~J}$, thickened, branched cellular processes) and perivascular infiltrates (Fig. 3K). Figure 3L highlights clusters of CD68 staining adjacent to an immunonegative balloon cell. Furthermore, there was strong CD68 staining in round inflammatory cells scattered through the adjacent subcortical white matter (Fig. 3M).

Cellular ABC staining was found in large roundish subpial cells (Fig. 3N, arrows; arrowheads point to additional, even subpial bandlike immunoreactivity to ABC). Similar cells were scattered throughout the dyslaminated cortical layers (Fig. 3O). Higher magnification revealed different staining morphologies (Fig. 3P and Q); balloon cells exhibited strong staining beside cells with a more bush-like appearance. Subcortical areas adjacent to dyslaminated cortical areas exhibited strong immunoreactivity to $\mathrm{ABC}$ in round cells and cells that exhibited a glial appearance (Fig. 3R, arrow).

As with cases of FCD Type IIA, immunoreactivity to CD34 was detected with vascular/endothelial staining patterns (Fig. 3S-W, small black arrows). However, lesional (MRI-positive) FCD Type IIB cases exhibited additional nonvascular cellular CD34 immunolabeling. Therefore, CD34-positive cells exhibited different morphological appearances; there were few bushlike cells scattered throughout dyslaminated cortical areas (Fig. 3S, arrowheads; Fig. $3 \mathrm{~S}$ and higher magnification in Fig. 3T; same cortical area as that shown in Fig. 3O). Some CD34 immunoreactivity appeared as surface staining around balloon cells (Fig. $3 \mathrm{U}$ and $\mathrm{V}$, arrowheads). In addition, there was widespread coral-like CD34 staining in dyslaminated cortical areas (Fig. 3W).

In general, this cellular CD34 staining was confined to specimens from patients with FCD Type IIB that had been confirmed by imaging. In the sole MRI-negative case of FCD Type IIB (Case 10), nonvascular CD34 staining was not exhibited.

\section{Discussion}

The aim of our study was to investigate potential distinctions between FCD Types IIA and IIB with a focus on 

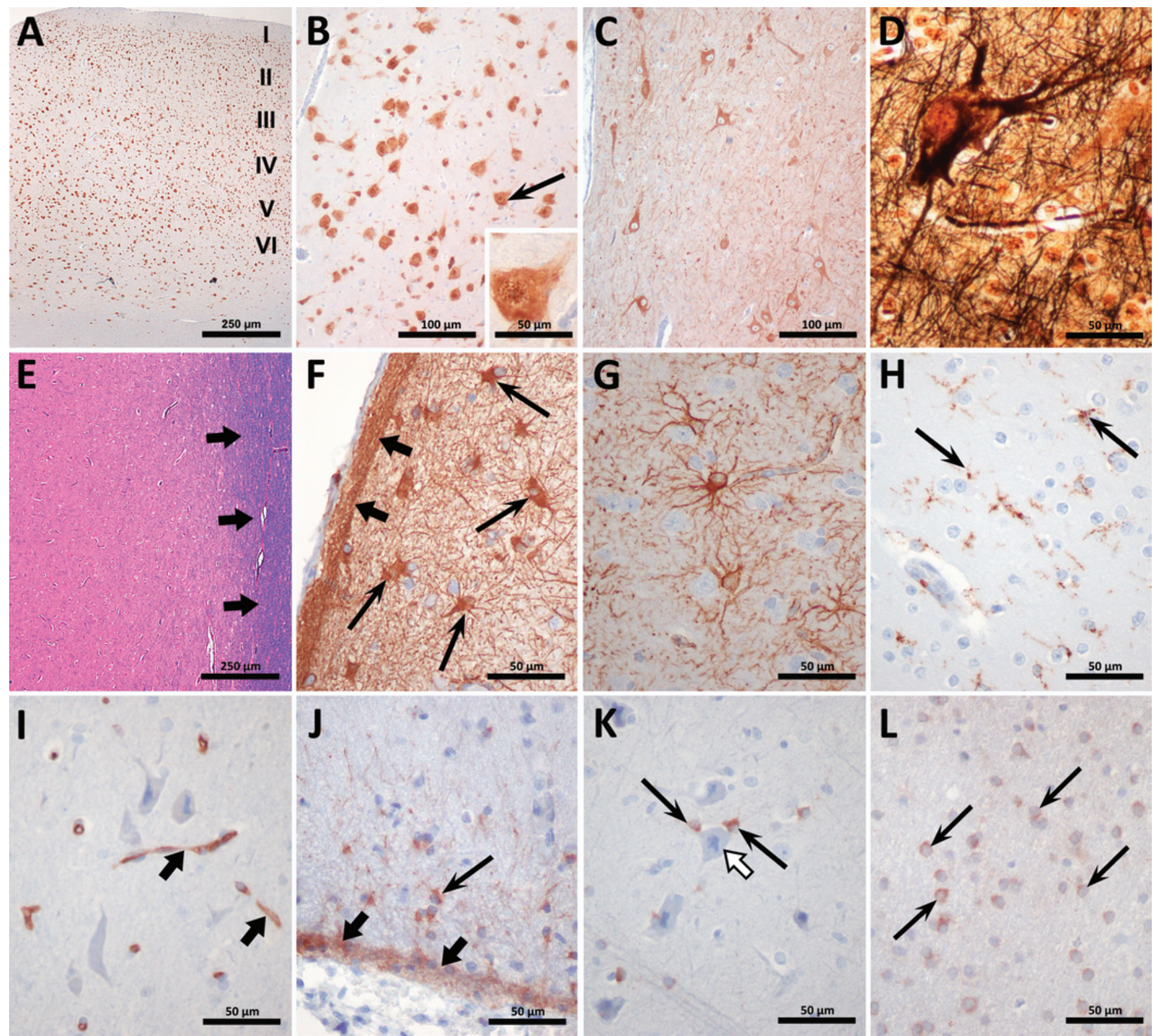

FIG. 2. Histochemical and immunohistochemical findings in FCD Type IIA cases. A: NeuN staining highlighting focal cortical dyslamination. B-D: NeuN (higher magnification of area in A), neurofilament, and Bielschowsky staining, respectively, showing characteristics of dysmorphic neurons. E: Intact gray-white matter junction in a specimen from a patient with FCD Type IIA (Case 5) (H \& E/LFB stain). F: GFAP staining revealed strong pial gliosis (Chaslin's gliosis) (thick arrows) and adjacent glial cells (thin arrows). G: GFAP-positive cells in dyslaminated cortical areas showing morphological features of cellular reactivity. H: CD68-stained microglial and inflammatory cells (arrows). I: In all cases of FCD Type IIA, CD34 staining was confined to vascular structures (arrows). J: Pial ABC staining (thick arrows) and adjacent ABC-positive cells (thin arrows). K and L: a-B crystallin staining shows few small round cells in dyslaminated cortical areas (K, black arrows) located beside ABC-negative cells with morphological features of dysplastic neurons (white arrow) and small round cells in the white matter ( $\mathbf{L}$, white matter area adjacent to dysplastic cortical area). Figure is available in color online only.

extratemporal epilepsy in children. By applying the 2011 ILAE classification system, we found 14 cases, 7 with extratemporal FCD Type IIA and 7 with extratemporal FCD Type IIB.

\section{Clinical Parameters}

In our series, the age of seizure onset was earlier in patients with FCD Type IIA than in those with FCD
Type IIB. In most cases, seizure onset occurred in early childhood, which is in accordance with data in the literature. ${ }^{16,22,43}$ Seizure frequencies and types were similar. Patients with FCD Type IIB underwent slightly more cortical resections than those with FCD Type IIA (6 in the FCD Type IIA group, 9 in the FCD Type IIB group).

Clinical outcome, when defined using Engel's classification, seemed better in patients with FCD Type IIA than 


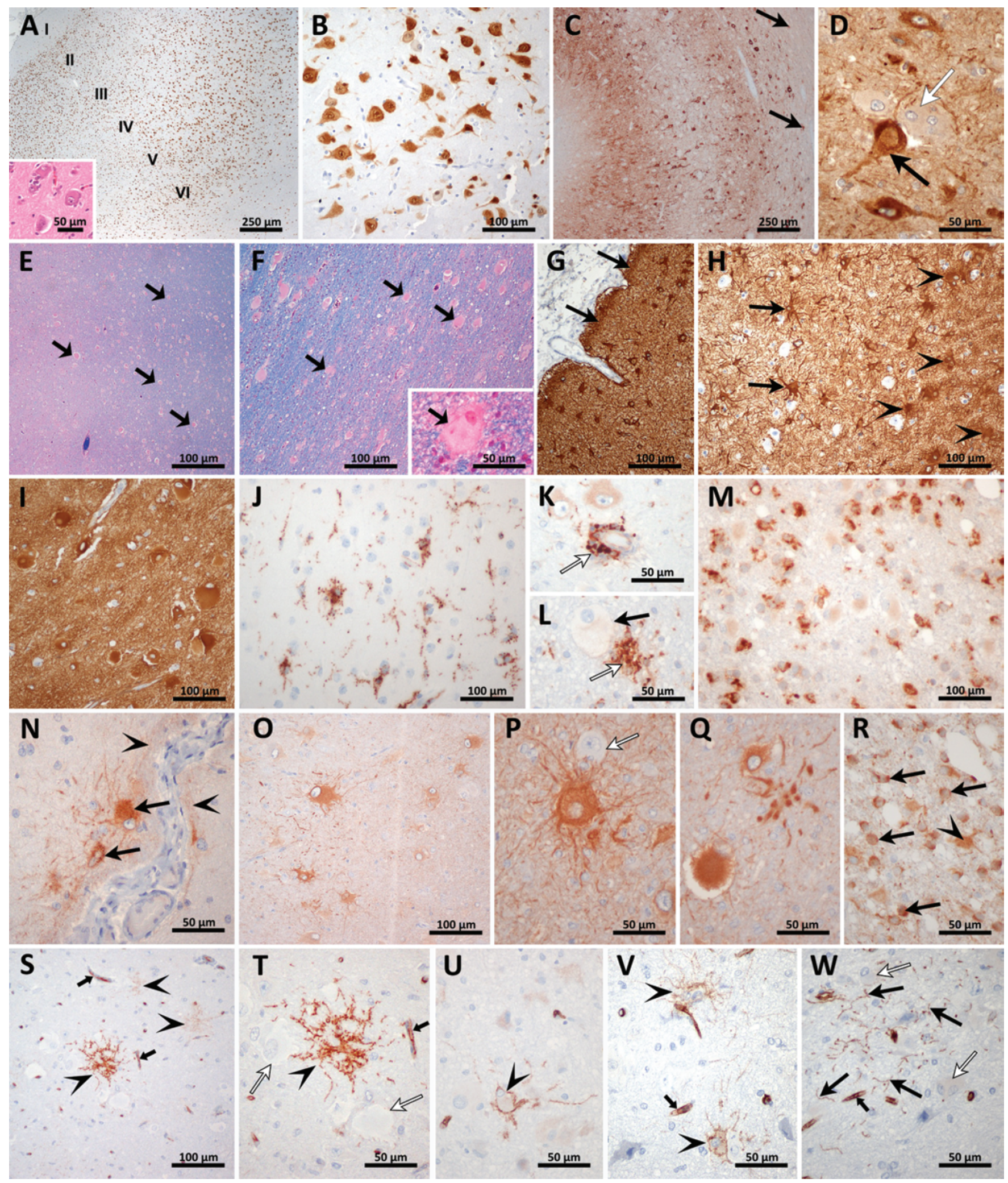

FIG. 3. Pathological features of MRI-positive FCD Type IIB cases. A and B: Focal cortical dyslamination with dysplastic NeuN-positive neurons scattered throughout all cortical layers. C: Maloriented NF-positive neuronal cells; some of these cells were found in the adjacent subcortical white matter (arrows). D: NF-positive dysplastic cells in close proximity to roundish NF-negative cells (white arrow, located close to dysplastic neurons [black arrow]). E and F: Staining with H \& E/LFB disturbed gray-white matter border by intermingled balloon cells (black arrows). Also shown are balloon cells in deeper cortical layers and the adjacent gray-white matter border ( $\mathbf{D}$ and $\mathbf{F}$ inset). $\quad$ FIG. 3. (continued) $\rightarrow$ 
FIG. 3. G and H: Strong GFAP staining in subpial (G, arrows), cortical areas ( $\mathbf{G}$ and $\mathbf{H})$, and gray-white matter junction (H). Shown also is intense immunoreactivity to GFAP in bushlike cells in the adjacent subcortical white matter ( $\mathbf{H}$, arrowheads) and GFAP-immunoreactive balloon cells (I). $\mathbf{J}$ and $\mathbf{K}$ : CD68 staining revealed microglial activation $(\mathrm{J})$ and perivascular infiltrates (K, white arrow). L: Exemplary example of bush-like CD68 staining (white arrow) adjacent to an immunonegative balloon cell (black arrow). M: In addition, strong CD68 staining scattered throughout the adjacent subcortical white matter was found. N-R: a-B crystallin staining in big, roundish subpial cells (N, arrows; arrowheads point to additional, even subpial bandlike immunoreactivity to $A B C)$. Similar cells were scattered throughout the dyslaminated cortical layers (0; higher magnification with different staining morphologies [P and Q]). Subcortical areas adjacent to dyslaminated cortical areas exhibited strong immunoreactivity to ABC in round cells resembling oligodendroglial cells (R, arrow) and cells with a microglial appearance (R, arrowhead). S-W: As with cases of FCD Type IIA, immunoreactivity to CD34 was detected with endothelial staining patterns (S, T, V, and W, small black arrows). Lesional (MRI-positive) FCD Type IIB cases exhibited additional nonendothelial cellular CD34 immunolabeling with bushlike cells scattered throughout dyslaminated cortical areas (S, arrowheads; higher magnification in T; identical cortical area as that shown in 0). Some immunoreactivity to CD34 appeared as surface staining around balloon cells (U and V, arrowheads). Coral-like CD34 staining in dyslaminated cortical areas adjacent to immunonegative cells (W, white arrows). Figure is available in color online only.

in those with Type IIB. However, in light of our relatively small case numbers, definite conclusions about the difference in outcome after resection for FCD Types IIA and IIB cannot be drawn. Furthermore, one has to consider additional aspects that can influence success rates of cortical resection, such as lesion extension and localization. Fauser et al. ${ }^{15}$ reported a significantly better surgical outcome in patients with unilobar FCD than in those with multilobar FCD (including FCD Types I and II).

In keeping with results of the Fauser et al. ${ }^{15}$ study, the less favorable Engel's outcome for the patients in Cases 4 and 14 of our series was most probably a result of lesions being more widespread than in the other patients with welldefined cortical lesions. For instance, cortical dysplasia in the Case 4 patient involved the left frontal and parietal lobes extending into the deep white matter, including the left internal capsule. Even after gross resection, also confirmed by postoperative MRI with no residual hyperintensities on T2-weighted/FLAIR images, ECoG still revealed sporadic spikes after resection. Furthermore, pathology that involved eloquent areas of the brain, where cortical resection is not feasible, is another important factor that determined surgical outcome. In the Case 7 patient, postresection ECoG revealed spikes in the motor cortex with residual precentral cortical areas of unusual morphology found in postoperative imaging. A similar situation occurred in Case 14 after the patient underwent resection for lesional epilepsy related to occipital brain areas. These three patients achieved an overall worse Engel's outcome score than the other patients in whom no residual postresection discharges were monitored. This result might be in concordance with the findings of Greiner et al., ${ }^{20}$ who viewed postresection discharges as a prognostic marker. Nonetheless, because of the small number of cases involved, our data cannot provide any significant relation between postresection ECoG or MRI results and clinical outcome in patients with lesional or nonlesional FCD-related epilepsy (especially against the background of discrepancy in the current literature on this topic with the results of El Tahry et al., ${ }^{12}$ who found no correlation between outcome and persistence of postresection epileptiform discharges). However, our findings support the notion that postoperative long-term outcome for FCD treatment is multifactorial, not determined only by histology but also the extent of the lesion and the achieved resection grade. ${ }^{15}$

Although we had only a small number of cases, their clinical presentations were similar to those described in the literature, where the clinical spectrum of FCD Type II is highly variable, and it is impossible to assign a typical disease pattern to this formal pathological entity. ${ }^{38}$ Therefore, clinical differentiation between FCD Types IIA and IIB is yet an unsolved problem, which gives rise to concern that distinction between both subgroups might need reconsideration in the future?

\section{Neuroimaging Features}

In our study on neuroimaging, T1-weighted MRI revealed cortical alteration in 3 of 7 patients with FCD Type IIA, whereas cortical pathologies were found in 6 of 7 with FCD Type IIB (cortical hyperintensities were seen in T2weighted/FLAIR images of 4 patients, and the transmantle sign was found in 2 patients). Thus, MRI alterations that are known to be typically associated with FCD (increased cortical thickness, blurred gray-white matter junction on T1- and T2-weighted images, increased signal on T2-weighted/FLAIR images, gyration anomalies, and the transmantle sign [FCD Type IIB]) were found in all except 1 of our FCD Type IIB cases. ${ }^{7,43,45}$ The transmantle sign, which is a white matter tail-like hyperintensity seen with T2-weighted imaging that spans from the affected cortex (usually crown or bottom of the gyrus) to the underlying ventricle, was found only in patients with FCD Type IIB. The underlying pathology is thought to be related to radial glial/neuronal unit involvement and is viewed as a typical imaging finding in cases of FCD Type IIB. ${ }^{3,5}$

Our findings mirror observations reported in the recent literature, in which more abnormal MRI results have been found in patients with FCD Type IIB than in those with FCD Type IIA. ${ }^{9,40}$ Blümcke et al. ${ }^{7}$ demonstrated a correlation between transmantle-sign T2-weighted/FLAIR hyperintensity and lack of normal myelin content in the respective FCD areas. Although we did not aim to quantify demyelination, all specimens from patients with FCD Type IIB exhibited blurring of the cortex-white matter border as a result of balloon cell clustering, especially in the subcortical areas of dysplastic cortical brain regions, a finding that is also related to T2-weighted/FLAIR hyperintensities on respective MRIs. ${ }^{40}$

It is interesting to note that the case with MRI-negative FCD Type IIB, in which no epilepsy-related cortical MRI changes were found, also differed from the other FCD Type IIB cases in respect to the CD34 immunohistochemical staining pattern (discussed in the next paragraph). 


\section{Neuropathological Findings in Extratemporal FCD Types IIA and IIB}

When the 2011 ILAE criteria on H \& E-stained sections were applied, typical FCD Type II-defining histopathological features were identifiable in 14 cases. Abnormal gross neurons reached an approximate median $( \pm \mathrm{SD})$ diameter of $58 \pm 22 \mu \mathrm{m}$ in patients with FCD Type IIA and $78 \pm 28 \mu \mathrm{m}$ in those with FCD Type IIB (normal pyramidal neurons in the midcortical layer diameter ranged from 12 to $25 \mu \mathrm{m})$. Otherwise, there were no obvious morphological differences between dysmorphic neuronal cells in FCD Type IIA and IIB cases, which is in accordance with data in the literature?

Balloon cells, the hallmark of FCD Type IIB, were present in 7 cases. With $\mathrm{H} \& \mathrm{E}$ staining, these cells exhibited typical histopathological features, such as large pale eosinophilic cell bodies and occasional multiple nuclei. These cells were found in all cortical layers (I-VI) and intermingled within the gray-white matter junction, which resulted in blurring with $\mathrm{H} \& \mathrm{E} / \mathrm{LFB}$ staining. Clusters of these cells were found in the adjacent subcortical white matter. In our immunohistochemical investigations, balloon cells exhibited immunoreactivity to GFAP and ABC. ${ }^{14,37,41}$ Distinct balloon cells exhibited filigree CD34 stain lining the cytoplasm and cellular processes, as described by Fauser et al. ${ }^{14}$ There were no CD68 or neuronal markers found to be expressed in these cells.

Because the clinical distinction between FCD Types IIA and IIB is unreliable or impossible (see previous discussion on clinical parameters), histopathological differentiation between these subgroups is paramount. Such differentiation might be difficult if surgical specimens are small or unclear in orientation, and such specimens might not be truly representative of the lesion. Therefore, identification of biomarkers might be a key element in differentiating between FCD Type II entities. In this regard, a widening spectrum of immunohistochemical and biological markers has been applied recently to assist with FCD classification. ${ }^{18,21,25,27,30,32,42}$ These markers might also provide clues for understanding the underlying pathogenesis. For example, Sakakibara et al. ${ }^{36}$ found differences in the expression patterns of the cortical layer-specific markers TBR1 and FOXP1 in FCD Types IIA and IIB. The authors concluded that FCD Type IIB is a more severe malformation with an earlier pathological onset than that of FCD Type IIA. ${ }^{36}$

In our study, $\mathrm{ABC}$ and CD34 were chosen as additional established immunohistochemical markers to further examine their expression patterns in specimens from patients with FCD Type IIA or IIB extratemporal epilepsy. The heat-shock protein $\mathrm{ABC}$ was investigated by Sarnat and Flores-Sarnat ${ }^{37}$ as a marker of epileptogenic foci in FCD and epilepsy-associated pathologies. In our study, ABC staining highlighted cells with glial morphology in the white matter, subpial glial cells, and satellite glial cells in patients with either of the FCD Type II subtypes. $\alpha$-B crystallin-immunoreactive cells in the white matter exhibited characteristics as described by Krishnan et al..$^{24}$ and had morphological attributes of both astrocytes and oligodendrocytes. The widespread glial immunoreactivity to $\mathrm{ABC}$, especially in the deep white matter, indicated what was interpreted to be a reactive rather than a specific pattern.
In addition, sections from patients with FCD Type IIB exhibited strong immunoreactivity to $\mathrm{ABC}$ in balloon cells, located in the white matter and gray-white matter border and as clusters in dyslaminated cortical areas. These staining patterns confirmed those described for FCD by Sarnat and Flores-Sarnat. ${ }^{37}$ Thus, aside from known balloon cell positivity with FCD Type IIB, ABC staining did not add any further information for distinguishing between FCD Types IIA and IIB in our study. However, in specimens with rare balloon cells or difficult section orientation, in which detection of theses cells with $\mathrm{H}$ \& $\mathrm{E}$ staining can be challenging, their conspicuous $\mathrm{ABC}$ positivity turned out to support confirmation of the diagnosis of FCD Type IIB rather than that of FCD Type IIA in our studies.

CD34 is a glycoprotein that is expressed on the surface of hematopoietic progenitor cells, on vascular endothelium, and in the CNS during early neurulation. Therefore, it has been viewed and used as a stem cell marker in the recent literature. ${ }^{4,8,26}$ In our studies, CD34 resulted in consistent vascular/endothelial staining patterns in both FCD Type II subgroups (IIA and IIB, respectively). As such, this endothelial CD34 staining served as an intrinsic positive control. In contrast, additional nonvascular cellular CD34 staining was found exclusively in dyslaminated cortical and adjacent white matter areas in patients with FCD Type IIB had lesions revealed by MRI that were typical for FCD, as described in detail above.

Different CD34 staining patterns, such as those with a netlike appearance or solitary, clustered, and diffuse staining patterns, have been described for glioneuronal lesions associated with epilepsy.,30 This cellular immunoreactivity to CD34 was thereby described for dyslaminated cortical regions that spanned from the subpial molecular layer to the cortical-white matter border, which corresponds to our own findings but contrasts descriptions of restricted expression of CD34 in balloon cells located in the deep white matter or gray-white matter boundary in cases of FCD Type IIB, ${ }^{14}$ because CD34-positive cells were detected also in more superficial cortical layers. However, the described membranous staining pattern with CD34 immunoreactivity coating the cell surface of balloon cells and spreading along their proximal cell processes was also apparent in our specimens from patients with FCD Type IIB. The nature of these CD34-positive cells, which appear beside immunonegative balloonlike cells, is still to be determined. To date, they have been discussed as potential dysplastic glial cells ${ }^{4}$ or indifferent cells with potency to differentiate into glial or neuronal cells (depending on their location within the white matter).$^{14}$ Regarding FCD, Deb et al. ${ }^{11}$ described CD34-immunoreactive cells as single cells or small cell clusters, which was also the case in our studies. Massive CD34-immunoreactive clusters, as described for temporal lesions associated with neoplasms (ganglioglioma), ${ }^{4}$ were not recognized in our investigation.

The function of CD34 is yet to be clarified; however, there have been hints to its involvement in cell adhesion and intracellular signal transduction pathways. ${ }^{23} \mathrm{Be}-$ side vascular (endothelial) expression in the adult CNS, CD34-positive cells have been described among others in Schwann cell tumors, tumors of the meninges, gangliogliomas, pleomorphic xanthoastrocytomas, and other cor- 
tical malformations associated with epilepsy., ${ }^{4,17,35}$ In the context of epilepsies, various neoplastic lesions in patients with chronic temporal lobe epilepsy exhibited strong immunoreactivity to CD34, which suggests a specific role for CD34 during temporal lobe development. ${ }^{4}$

Only a few small studies also investigated CD34 expression in relation to FCD Type I or II. ${ }^{11,14,30,39}$ The findings of Deb et al. ${ }^{11}$ regarding cellular CD34 staining in specimens from patients with FCD differed from our and others' observations. Of their 3 (of 18) cases of CD34-positive FCD, none were from specimens from patients with FCD Type IIB. ${ }^{11}$ They also noted that these cases lacked immunoreactivity to CD34 in balloon cells, which again is contrary to our findings and those of others. ${ }^{4,17}$ These differences are difficult to reconcile based on their case numbers (2 cases of FCD Type IIB), different brain regions, and the possibility of differences in methodology (especially regarding tissue sampling).

With our studies we were able to demonstrate consistent cellular immunoreactivity to CD34 in specimens from patients with FCD Type IIB, which was confined to all "lesional" (i.e., MRI-positive) cases. CD34 positivity can be viewed essentially as another marker of FCD Type IIB and can serve as further evidence that the etiology and biology of FCD Types IIA and IIB might be different despite them being grouped together. Because there is similar positivity in many low-grade epileptogenic tumors, FCD Type IIB might be related more closely to these tumors than FCD Type IIA.

Additional investigations are needed to clarify whether these differences in immunoreactivity to CD34 has functional significance regarding pathogenesis or etiology of FCD Types IIA and IIB. CD34 immunohistochemistry provided a valuable diagnostic tool in our studies to confirm and differentiate the diagnosis of FCD Type IIB, as was $\mathrm{ABC}$ staining in anatomically difficult arrangements.

\section{Acknowledgments}

We thank Dr. Sargent for providing initial and postoperative MRI reports. Dr. Knerlich-Lukoschus received a travel stipend from the German Society of Neurosurgery (DGNC).

\section{References}

1. Aronica E, Becker AJ, Spreafico R: Malformations of cortical development. Brain Pathol 22:380-401, 2012

2. Avoli M, Bernasconi A, Mattia D, Olivier A, Hwa GG: Epileptiform discharges in the human dysplastic neocortex: in vitro physiology and pharmacology. Ann Neurol 46:816826, 1999

3. Barkovich AJ, Kuzniecky RI, Bollen AW, Grant PE: Focal transmantle dysplasia: a specific malformation of cortical development. Neurology 49:1148-1152, 1997

4. Blümcke I, Giencke K, Wardelmann E, Beyenburg S, Kral T, Sarioglu N, et al: The CD34 epitope is expressed in neoplastic and malformative lesions associated with chronic, focal epilepsies. Acta Neuropathol 97:481-490, 1999

5. Blümcke I, Mühlebner A: Neuropathological work-up of focal cortical dysplasias using the new ILAE consensus classification system - practical guideline article invited by the Euro-CNS Research Committee. Clin Neuropathol 30:164177,2011

6. Blümcke I, Spreafico R: An international consensus classifi- cation for focal cortical dysplasias. Lancet Neurol 10:26-27, 2011

7. Blümcke I, Thom M, Aronica E, Armstrong DD, Vinters HV, Palmini A, et al: The clinicopathologic spectrum of focal cortical dysplasias: a consensus classification proposed by an ad hoc Task Force of the ILAE Diagnostic Methods Commission. Epilepsia 52:158-174, 2011

8. Brown J, Greaves MF, Molgaard HV: The gene encoding the stem cell antigen, CD34, is conserved in mouse and expressed in haemopoietic progenitor cell lines, brain, and embryonic fibroblasts. Int Immunol 3:175-184, 1991

9. Colombo N, Tassi L, Deleo F, Citterio A, Bramerio M, Mai $\mathrm{R}$, et al: Focal cortical dysplasia type IIa and IIb: MRI aspects in 118 cases proven by histopathology. Neuroradiology 54:1065-1077, 2012

10. Crino PB, Eberwine J: Cellular and molecular basis of cerebral dysgenesis. J Neurosci Res 50:907-916, 1997

11. Deb P, Sharma MC, Tripathi M, Sarat Chandra P, Gupta A, Sarkar C: Expression of CD34 as a novel marker for glioneuronal lesions associated with chronic intractable epilepsy. Neuropathol Appl Neurobiol 32:461-468, 2006

12. El Tahry R, Ferrao Santos S, de Tourtchaninoff M, Géraldo Ribeiro Vaz J, Finet P, Raftopoulos C, et al: Post-resection electrocorticography has no added value in epilepsy surgery. Acta Neurol Belg 116:279-285, 2016

13. Engel J, Van Ness PC, Rasmussen TB, Ojemann LM: Outcome with respect to epileptic seizures, in Engel J Jr (ed): Surgical Treatment of the Epilepsies. New York: Raven Press, 1993

14. Fauser S, Becker A, Schulze-Bonhage A, Hildebrandt M, Tuxhorn I, Pannek HW, et al: CD34-immunoreactive balloon cells in cortical malformations. Acta Neuropathol 108:272278, 2004

15. Fauser S, Essang C, Altenmüller DM, Staack AM, Steinhoff BJ, Strobl K, et al: Long-term seizure outcome in 211 patients with focal cortical dysplasia. Epilepsia 56:66-76, 2015

16. Fauser S, Huppertz HJ, Bast T, Strobl K, Pantazis G, Altenmueller DM, et al: Clinical characteristics in focal cortical dysplasia: a retrospective evaluation in a series of 120 patients. Brain 129:1907-1916, 2006

17. Fauser S, Schulze-Bonhage A, Honegger J, Carmona H, Huppertz HJ, Pantazis G, et al: Focal cortical dysplasias: surgical outcome in 67 patients in relation to histological subtypes and dual pathology. Brain 127:2406-2418, 2004

18. Foresti ML, Arisi GM, Shapiro LA: Role of glia in epilepsyassociated neuropathology, neuroinflammation and neurogenesis. Brain Res Brain Res Rev 66:115-122, 2011

19. Gaitanis JN, Donahue J: Focal cortical dysplasia. Pediatr Neurol 49:79-87, 2013

20. Greiner HM, Horn PS, Tenney JR, Arya R, Jain SV, Holland $\mathrm{KD}$, et al: Should spikes on post-resection ECoG guide pediatric epilepsy surgery? Epilepsy Res 122:73-78, 2016

21. Han CW, Min BW, Kim Y, Jeong EH, Park CS, Woo YJ, et al: Immunohistochemical analysis of developmental neural antigen expression in the balloon cells of focal cortical dysplasia. J Clin Neurosci 18:114-118, 2011

22. Harvey AS, Cross JH, Shinnar S, Mathern GW: Defining the spectrum of international practice in pediatric epilepsy surgery patients. Epilepsia 49:146-155, 2008 (Erratum in Epilepsia 54:1140, 2013)

23. Healy L, May G, Gale K, Grosveld F, Greaves M, Enver T: The stem cell antigen CD34 functions as a regulator of hemopoietic cell adhesion. Proc Natl Acad Sci U S A 92:1224012244, 1995

24. Krishnan B, Armstrong DL, Grossman RG, Zhu ZQ, Rutecki PA, Mizrahi EM: Glial cell nuclear hypertrophy in complex partial seizures. J Neuropathol Exp Neurol 53:502-507, 1994

25. Lim KC, Crino PB: Focal malformations of cortical develop- 
ment: new vistas for molecular pathogenesis. Neuroscience 252:262-276, 2013

26. Lin G, Finger E, Gutierrez-Ramos JC: Expression of CD34 in endothelial cells, hematopoietic progenitors and nervous cells in fetal and adult mouse tissues. Eur J Immunol 25:15081516,1995

27. Miles L, Greiner HM, Miles MV, Mangano FT, Horn PS, Leach JL, et al: Interaction between akt1-positive neurons and age at surgery is associated with surgical outcome in children with isolated focal cortical dysplasia. J Neuropathol Exp Neurol 72:884-891, 2013

28. Morioka T, Nishio S, Ishibashi H, Muraishi M, Hisada K, Shigeto H, et al: Intrinsic epileptogenicity of focal cortical dysplasia as revealed by magnetoencephalography and electrocorticography. Epilepsy Res 33:177-187, 1999

29. Mühlebner A, Coras R, Kobow K, Feucht M, Czech T, Stefan $\mathrm{H}$, et al: Neuropathologic measurements in focal cortical dysplasias: validation of the ILAE 2011 classification system and diagnostic implications for MRI. Acta Neuropathol 123:259-272, 2012

30. Nagaishi M, Arai M, Osawa T, Yokoo H, Hirato J, Yoshimoto Y, et al: An immunohistochemical finding in glioneuronal lesions associated with epilepsy: the appearance of nestinpositive, CD34-positive and tau-accumulating cells. Neuropathology 31:468-475, 2011

31. Najm IM, Tassi L, Sarnat HB, Holthausen H, Russo GL: Epilepsies associated with focal cortical dysplasias (FCDs). Acta Neuropathol 128:5-19, 2014

32. Nakashima M, Saitsu H, Takei N, Tohyama J, Kato M, Kitaura H, et al: Somatic mutations in the MTOR gene cause focal cortical dysplasia type IIb. Ann Neurol 78:375-386, 2015

33. Palmini A, Najm I, Avanzini G, Babb T, Guerrini R, Foldvary-Schaefer N, et al: Terminology and classification of the cortical dysplasias. Neurology 62 (6 Suppl 3):S2-S8, 2004

34. Raymond AA, Fish DR, Sisodiya SM, Alsanjari N, Stevens JM, Shorvon SD: Abnormalities of gyration, heterotopias, tuberous sclerosis, focal cortical dysplasia, microdysgenesis, dysembryoplastic neuroepithelial tumour and dysgenesis of the archicortex in epilepsy. Clinical, EEG and neuroimaging features in 100 adult patients. Brain 118:629-660, 1995

35. Reifenberger G, Kaulich K, Wiestler OD, Blümcke I: Expression of the CD34 antigen in pleomorphic xanthoastrocytomas. Acta Neuropathol 105:358-364, 2003

36. Sakakibara T, Sukigara S, Saito T, Otsuki T, Takahashi A, Kaneko Y, et al: Delayed maturation and differentiation of neurons in focal cortical dysplasia with the transmantle sign: analysis of layer-specific marker expression. J Neuropathol Exp Neurol 71:741-749, 2012

37. Sarnat HB, Flores-Sarnat L: Alpha-B-crystallin as a tissue marker of epileptic foci in paediatric resections. Can J Neurol Sci 36:566-574, 2009

38. Sisodiya SM, Fauser S, Cross JH, Thom M: Focal cortical dysplasia type II: biological features and clinical perspectives. Lancet Neurol 8:830-843, 2009

39. Sung CO, Suh YL, Hong SC: CD34 and microtubule-associated protein 2 expression in dysembryoplastic neuroepithelial tumours with an emphasis on dual expression in non-specific types. Histopathology 59:308-317, 2011

40. Tassi L, Garbelli R, Colombo N, Bramerio M, Russo GL, Mai R, et al: Electroclinical, MRI and surgical outcomes in 100 epileptic patients with type II FCD. Epileptic Disord 14:257-266, 2012

41. Urbach H, Scheffler B, Heinrichsmeier T, von Oertzen J, Kral T, Wellmer J, et al: Focal cortical dysplasia of Taylor's balloon cell type: a clinicopathological entity with characteristic neuroimaging and histopathological features, and favorable postsurgical outcome. Epilepsia 43:33-40, 2002

42. Wang VY, Chang EF, Barbaro NM: Focal cortical dysplasia: a review of pathological features, genetics, and surgical outcome. Neurosurg Focus 20(1):E7, 2006

43. Widdess-Walsh P, Kellinghaus C, Jeha L, Kotagal P, Prayson $\mathrm{R}$, Bingaman W, et al: Electro-clinical and imaging characteristics of focal cortical dysplasia: correlation with pathological subtypes. Epilepsy Res 67:25-33, 2005

44. Wieser HG, Blume WT, Fish D, Goldensohn E, Hufnagel A, King D, et al: ILAE Commission Report. Proposal for a new classification of outcome with respect to epileptic seizures following epilepsy surgery. Epilepsia 42:282-286, 2001

45. Woermann FG, Vollmar C: Clinical MRI in children and adults with focal epilepsy: a critical review. Epilepsy Behav 15:40-49, 2009

\section{Disclosures}

The authors report no conflict of interest concerning the materials or methods used in this study or the findings specified in this paper.

\section{Author Contributions}

Conception and design: Knerlich-Lukoschus, Connolly, Steinbok, Dunham. Acquisition of data: Knerlich-Lukoschus, Hendson. Analysis and interpretation of data: Knerlich-Lukoschus, Dunham. Drafting the article: Knerlich-Lukoschus. Critically revising the article: all authors. Reviewed submitted version of manuscript: all authors. Approved the final version of the manuscript on behalf of all authors: Knerlich-Lukoschus. Administrative/ technical/material support: Connolly, Dunham. Study supervision: Knerlich-Lukoschus, Connolly, Steinbok, Dunham.

\section{Correspondence}

Friederike Knerlich-Lukoschus, Department of Neurosurgery, University Hospital of Schleswig-Holstein Campus Kiel, ArnoldHeller-Strasse 3, House 41, Kiel 24105, Germany. email: friederike.knerlich@uksh.de. 